\title{
Corpus
}

\section{El estudio de la guerra en la arqueología sur-andina}

\author{
Axel E. Nielsen
}

\section{(2) OpenEdition}

\section{Journals}

Electronic version

URL: http://journals.openedition.org/corpusarchivos/1393

DOI: 10.4000/corpusarchivos. 1393

ISSN: 1853-8037

\section{Publisher}

Diego Escolar

\section{Electronic reference}

Axel E. Nielsen, « El estudio de la guerra en la arqueología sur-andina », Corpus [En línea], Vol 5, No 1 | 2015, Publicado el 30 junio 2015, consultado el 08 mayo 2020. URL : http://journals.openedition.org/ corpusarchivos/1393; DOI : https://doi.org/10.4000/corpusarchivos.1393

This text was automatically generated on 8 May 2020.

Licencia Creative Commons: Atribución-NoComercial 2.5 Argentina (CC BY-NC 2.5 AR) 


\title{
El estudio de la guerra en la arqueología sur-andina
}

\author{
Axel E. Nielsen
}

1 Si entendemos la guerra como hostilidad armada entre colectivos políticamente organizados, las evidencias materiales indican que esta ha rondado a la humanidad durante gran parte de su historia, aunque con significativas variaciones de intensidad y forma. A pesar de su ubicuidad, la guerra recién se instala como un tema de investigación significativo en la arqueología -fundamentalmente en la anglosajonaen la década de 1990 (Kelly 1996). En los Andes todavía es un tópico escasamente discutido, a pesar de que las tradiciones orales recopiladas al momento de la conquista española aludían claramente a una era de conflictos armados antes de la expansión inca (Cieza de León 1984 [1553], Wuaman Puma 1980 [1615], entre otros) y deque los arqueólogos advirtieron hace tiempo ya sus rastros materiales (p. ej., Casanova 1936, Madrazzo y Ottonello 1966).

2 A continuación paso revista al estado actual de la reflexión arqueológica sobre las guerras prehispánicas andinas, particularmente las de época pre-inca en los Andes del Sur (NO de Argentina, $\mathrm{N}$ de Chile, SO de Bolivia). Siguiendo los ejes planteados para este intercambio de ideas, comienzo señalando los principales temas en debate, después trato las posibles consecuencias sociales del conflicto y termino considerando brevemente el modo en que se recordaban aquellos conflictos en la época colonial.

\section{Acuerdos y desacuerdos en torno a las guerras prehispánicas sur-andinas}

3 Cuatro clases de indicadores son comúnmente aceptados en arqueología para inferir la existencia de guerras, a saber, sistemas de asentamiento defensivos, señales de traumas en los cuerpos, artefactos vinculados al combate e iconografía (LeBlanc 1999, pp. 54-91). Si bien existen evidencias aisladas de épocas anteriores (p. ej., huesos humanos con traumas o puntas de proyectil incrustadas o representaciones de individuos con atuendos guerreros), es recién a partir del 1000 d.C. -y particularmente después de 
1200- que los cuatro tipos de indicadores se hacen presentes en gran parte del sur andino (Arkush 2006, Arkushy Tung 2013,Balesta y Wynveldt 2009, Berenguer 2006, Gheggi y Seldes 2012,Nielsen 2002, 2003, Torres Rouff et al. 2005, Williams 2014a). El clima de beligerancia sugerido por estas evidencias parece haber cesado con la expansión inca (la Pax Inca), teniendo en cuenta que muchos asentamientos defensivos dejan de utilizarse en el siglo XV y se establecen sitios nuevos en lugares claramente vulnerables (Nielsen 2002, Williams 2014b). Las fuentes documentales sugieren que los incas hicieron un uso restringido de la violencia armada, solo para doblegar la resistencia de algunos grupos (p. ej., collas, chichas) o defender la frontera oriental.

El primer tema que divide la opinión de los arqueólogos es si estos elementos son suficientes para concluir que efectivamente hubo guerras antes del inca, una época que en las secuencias cronológicas del área se denomina Período de Desarrollos Regionales o Intermedio Tardío (en adelante PDR). Los escépticos suelen cuestionar la validez de cada indicador por separado, argumentando por ejemplo que las cabezas cercenadas son un ejemplo más de la popularidad del culto a los antepasados; que las representaciones de guerreros o combates en el arte rupestre podrían aludir a situaciones míticas; que los cascos y corazas confeccionados en madera, fibra y cuero no parecen ser protección suficiente en combate; que las nuevas armas pudieron estar destinadas a la caza o -en el caso de hachas y manoplas- ser objetos meramente rituales; que muchos poblados de la época no tienen fortificaciones por lo que no son estrictamente pukaras y que su ubicación en puntos elevados pudo obedecer a consideraciones utilitarias (p. ej., maximizar el aprovechamiento de tierras cultivables o resguardarse de los aluviones) o religiosas.

5 Lo primero que esta forma de razonamiento ad hoc no puede explicar es porqué estas evidencias proliferan en forma simultánea (desde el punto de vista arqueológico) sobre un área tan extensa (desde la provincia de Catamarca, por lo menos, hasta el lago Titicaca y la sierra del Perú). Ciertamente, no aparecen en todas partes ni con la misma claridad y rara vez se encuentran todas ellas en una misma región. De hecho, no parece haber rastros de conflicto en las zonas más elevadas de la puna y altiplano, habitadas por pastores especializados (sureste de Lípez, puna occidental de Jujuy y Salta), ni entre los pescadores del litoral del océano Pacífico ${ }^{1}$. Cuando están, se asocian a poblaciones que practican la agricultura, tanto en los bolsones altiplánicos (p. ej., Antofagasta de la Sierra, Casabindo, Norte de Lípez, intersalar) como en las quebradas, valles y oasis a ambos lados del macizo cordillerano (p. ej., San Pedro de Atacama, Loa medio y superior, valles altos de Tarapacá al occidente, Valle de Hualfín, Valles Calchaquíes, Quebradas del Toro y Humahuaca, Río Grande de San Juan al oriente). Volveré sobre la importancia de tener en cuenta este patrón espacial y temporal al momento de considerar la naturaleza del conflicto, sus causas y consecuencias.

6 No es posible aquí analizar cada uno de estos cuestionamientos (ver Arkush y Stanish 2005,Nielsen 2003), por lo que solo tomaré dos ejemplos: la interpretación de los asentamientos y la relación entre rito y conflicto. La adopción generalizada entre los agricultores sur-andinos de un sistema de asentamiento defensivo es a mi juicio la evidencia más contundente del establecimiento de un "estado de guerra endémica" en el siglo XIII. Limitar el problema a una cuestión tipológica - ¿es correcto clasificar los poblados de la época como fortalezas o pukaras?- lleva a perder de vista las demás propiedades defensivas de las nuevas estrategias de uso del espacio, como el dominio visual del entorno que permite prevenir asaltos sorpresivos, las ventajas defensivas que 
ofrecen terrenos altos $y$ escarpados aun cuando no exista una arquitecturaespecializada, el amparo de numerosos vecinos que brinda la residencia en grandes conglomerados, la intervisibilidad que permite advertir y movilizar rápidamente a aliados cercanos o el abandono de áreas para crear zonas de amortiguación, entre otras (Balesta y Wynveldt 2010, Williams 2014). Puesto que la etnografía indica que la sorpresa y el número de combatientes en cada bando son factores decisivos en la llamada "guerra tribal" (Ferguson 1995, Keely 1996, Redmond 1994, Wiessner y Tumu 1998, entre otros) cabe pensar que los recaudos defensivos señalados fueron tan o más importantes que la presencia de fortificaciones ${ }^{2}$. Por otra parte ¿cómo explicar si no la adopción generalizada de estas formas de habitar que implicaron dejar antiguas áreas de vivienda en favor del hacinamiento en sitios generalmente desprovistos de agua, poner distancia con las áreas de cultivo y pastoreo y abandonar zonas ricas en recursos que habían sido regularmente habitadas hasta entonces?

7 Ciertamente, ninguno de estos cambios en los asentamientos puede ser explicado por quienes consideran que los conflictos eran solo combates rituales análogos al t'inku etnográfico (Topic y Topic 1997, 2009). Aunque estas prácticas provocan heridos y hasta muertos, lo que permite caracterizarlas como "ritos" y no "guerras" es, precisamente, que no representan una amenaza para quienes no participan directamente, por lo que no llevan a las comunidades involucradas a adoptar recaudos defensivos. La guerra siempre conlleva actos rituales, lo que no significa que sea menos violenta o amenazadora, sino simplemente que quienes combaten aprovechan todos los recursos a su disposición, incluyendo hechizos, música, danzas, plantas alucinógenas y otras técnicas para movilizar el auxilio de ancestros, animales tutelares y otras agencias no humanas (Nielsen 2007). Los antiguos andinos también empleaban el rito para propiciar las cosechas, la multiplicación del ganado o la construcción de sus casas, lo que no convierte a su agricultura, ganadería y arquitectura en actividades "meramente" rituales.

8 Para quienes creen en la realidad de las guerras pre-incas se abre un segundo campo de discusión en torno a las especificidades de aquellas prácticas. ¿Quiénes se enfrentaban? ¿Cómo eran aquellos conflictos? ¿Qué normas los regulaban? ¿Cómo afectaban a otras actividades, como la agricultura o el tráfico interregional? Como estas indagaciones se encuentran en sus comienzos, solo mencionaré ejemplos de las diversas respuestas que comienzan a surgir. Respecto a las partes enfrentadas, se ha planteado que podría tratarse de conflictos locales entre vecinos, grupos del oriente amenazando a las poblaciones de tierras altas (un fenómeno del que hay testimonio escrito en época colonial) o luchas entre colectivos regionales (Nielsen 2003, Wynveldt y Balesta 2009). La ventaja de esta última interpretación reside en que su carácter multiplicador podría dar cuenta de la gran difusión y relativa contemporaneidad de los conflictos y, particularmente, de su presencia en la vertiente occidental andina, lejos del alcance que uno atribuiría a los ataques de grupos de las tierras bajas del oriente. La ausencia de fuentes de agua en la mayoría de los poblados, por su parte, ratifica que los combates debieron ser fundamentalmente emboscadas, asaltos sorpresivos y saqueos, antes que asedios o campañas orientadas a la conquista territorial, prácticas que estarían fuera de las capacidades logísticas de los pueblos de la época. La separación entre áreas residenciales y productivas (campos con terrazas y riego, ganadería trashumante) sugieren la existencia de normas capaces de regular la estacionalidad de las luchas, con temporadas de paz dedicadas a la producción (seguramente el verano) y épocas para la 
guerra. Esto podría también explicar la continuidad del tráfico interregional a pesar de las hostilidades, aunque hay otros escenarios posibles; por ejemplo, pudo haber grupos neutrales capaces de viajar e intercambiar a pesar de las tensiones (p. ej., pastores o costeros que no acusan indicios de conflicto en sus territorios), pero también ciclos que alternaran guerras con intercambios asociados a las negociaciones de paz como ha sido documentado etnográficamente en otras partes del mundo (p. ej., Wiessner 2009).

Otro tema en debate se refiere a las causas de las hostilidades. Las hipótesis propuestas incluyen presión demográfica y competencia por tierras, luchas políticas relacionadas con la disolución de Tiwanaku, contiendas por el control del tráfico de larga distancia, invasiones de otros grupos y deterioro climático (Arkush 2008, Balesta y Wynveldt 2010,Hyslop 1977, Núñez 1974, Ruiz y Albeck 1997,Schiappaccasse et al. 1989, ver evaluación en Nielsen 2003, pp. 95-97).La existencia de sequías en el sur andino durante el siglo XIV, que podrían haber abonado el clima de beligerancia, ha sido recientemente confirmada mediante reconstrucciones paleoclimáticas de alta resolución basadas en anillos de árboles (Morales et al. 2012). Cuando ocurren en la actualidad sequías de esta magnitud, conllevan el fracaso de la agricultura de secano en los sectores más áridos del altiplano, obligando a muchos hombres adultos a migrar a los valles y centros urbanos en busca de trabajo para sustentar a sus familias. Cabe pensar que crisis similares en el pasado no dejaron a las comunidades en las tierras altas más alternativas que apelar a la solidaridad de otros grupos menos afectados o presionar sobre sus vecinos a ambos lados de los Andes para asegurarse tierras con potencial para el riego (Llagostera 2010,Nielsen 2001a, Rothammer y Santoro 2001).

También comienza a cuestionarse recientemente el manejo de la fuerza militar y otras formas de violencia por parte de los incas. Las investigaciones de los últimos años han revelado que la formación del Tawantinsuyu en el siglo XV coincide con la destrucción violenta de áreas públicas y poblados importantes de varias regiones (Norte de Lípez, Loa superior, Río Grande de San Juan, Quebrada de Humahuaca), el abandono de ciertos asentamientos y la reorganización de otros, así como el vaciamiento de algunas regiones. Todo esto invita a revisar críticamente el supuesto de una expansión incaica negociada o impuesta pero sin uso efectivo de la fuerza, una idea basada en última instancia en testimonios orales recogidos durante la conquista que, sesgados por la voluntad propagandista del propio estado cuzqueño, pudieron haber omitido selectivamente ciertos hechos. Aunque con la conquista inca dejan de utilizarse sitios defensivos en ciertas regiones (p. ej., Norte de Lípez), en otras los sistemas de asentamiento locales no sufren cambios significativos, mientras que el Estado mismo parece controlar directamente las áreas de amortiguación o 'tierras de nadie' creadas durante el PDR mediante la construcción de tambos, guarniciones e instalaciones productivas.

\section{Las consecuencias sociales de las guerras pre- incaicas}

El escepticismo entre los arqueólogos respecto a la realidad o gravedad de las guerras pre-incaicas ha llevado a que, aunque a menudo se mencione al conflicto como una característica más de la época, se haya prestado poca atención a las consecuencias que pudo tener esta situación para los procesos sociales sur-andinos. El tema ha sido bastante discutido, en cambio, en la arqueología en general, principalmente con 
relación al papel que pudo desempeñar la guerra en el desarrollo de la complejidad social y, eventualmente, en el surgimiento del Estado (Carneiro 1970, Earle 1997, Haas 2001). Las opiniones varían ampliamente, pero últimamente hay cierto consenso en considerarla un elemento más capaz de promover cambios en las estructuras políticas y económicas, pero cuyos efectos específicos dependen de muchos otros factores cuya incidencia debe establecerse mediante un análisis histórico pormenorizado.

Hay elementos para afirmar que los conflictos del PDR provocaron cambios significativos en las formaciones sociales sur-andinas. En primer lugar, la concentración de la población en grandes asentamientos y la aparición en algunas regiones de relaciones jerárquicas entre ellos ponen de manifiesto procesos de integración que seguramente se asocian con nuevas prácticas e instituciones políticas, al menos entre los grupos agricultores y agro-pastores. Estas se hacen visibles en la proliferación de monumentos a los antepasados, los rastros de comensalismo público y la construcción de plazas en los principales poblados (Nielsen 2006). Todo indica que las nuevas pautas de convivencia y las alianzas entre grupos de parentesco y comunidades promovidas por la inseguridad resultaron en procesos de "fusión segmentaria" (Platt 1987) que resolvieron pacíficamente las disputas locales, redirigiendo la violencia hacia grupos ajenos a cada región. De acuerdo a esta interpretación, integración segmentaria local y guerra interregional se reforzarían mutuamente.

Tendencias hacia la intensificación e integración económica se advierten en el desarrollo de extensas superficies agrícolas con terrazas y andenes alimentados por complejos sistemas de irrigación que requieren una operación coordinada, como así también en la reorganización de los sistemas de movilidad y manejo del ganado. Más aún, podría argumentarse que el desarrollo de estructuras políticas supra-comunitarias estuvo acompañado de la implementación de estrategias económicas colectivas, en las que la formulación de los programas de trabajo, la planificación de uso de los recursos y otras decisiones económicas claves recaerían en manos de grupos o instancias administrativas superiores a la unidad doméstica. Por cierto, no tiene por qué haber una relación directa entre integración y desigualdad económica, entendida como participación diferencial en los procesos de trabajo y apropiación de recursos. La homogeneidad de la arquitectura doméstica y de los artefactos de uso cotidiano en general sugieren que la cultura material operaba como un discurso igualitario e inclusivo que sería consistente con un modo corporativo de acción política, pero la realidad es que no contamos aún con los datos de consumo necesarios para evaluar las desigualdades efectivas que pudieron existir en distintas regiones.

Otra tendencia notable en la cultura material del período es el desarrollo de estilos regionales diferenciados en la arquitectura, las inhumaciones, la vestimenta, la vajilla, las armas y otros elementos ubicuos en la experiencia cotidiana. Si este patrón revela el surgimiento de nuevas identidades colectivas, podríamos pensar que la guerra también favoreció procesos de etnogénesis, en los que afinidades culturales ancladas en una historia común y en la interacción local se acentuaron y asumieron carácter emblemático, diferenciando posibles grupos aliados de otrosy potenciales enemigos. Resulta sugerente que haya cierta correspondencia entre la ubicación de estos estilos y la de "grupos étnicos" que los conquistadores europeos documentaron en el siglo XVI. Ciertamente, la invención de tradiciones comúnmente asociada a la formación de etnicidades se vería facilitada por el masivo desarraigo y pérdida de memoria práctica que indudablemente debió acompañar a la relocalización de poblaciones del siglo XIII. 
La proliferación de referencias monumentales a los antepasados que se produce a partir de este momento (chullpas, wankas, etc.) es una expresión elocuente de este proceso político de reconfiguración de la memoria.

\section{Guerra y memoria}

15 Según los testimonios recogidos por los conquistadores españoles en el siglo XVI, la formación del Tawantinsuyu trajo consigo paz, mejoras económicas y un orden social justo, poniendo fin al caos ("behetrías") y a las guerras de la época anterior. Más allá del evidente sesgo ideológico que pesa sobre estas versiones "oficiales" pro-incas, resulta interesante cómo recordaban ese pasado guerrero los propios andinos. Considérese por ejemplo el relato que hace Waman Puma de Ayala sobre la edad de los guerreros (awca runas) anterior al inca:

Y se quitauan a sus mugeresy hijos y se quitauan sus sementeras y chacarasyasecyas de agua y pastos. Y fueron muy crueles que se rrobaron sus haziendas, rropa, plata, oro, cobre, hasta lleualle las piedras de moler que ellos les llaman maray, tonay, muchoca, callota, y belicosos yndios y traydores. Y tenían mucho oro y plata, puroncullque[plata nativa], puroncuri[oro nativo], y tenía muy mucha...

Y se hizieron grandes capitanes y ualerososprínzepes de puro uallente. Dizen que ellos se tornauan en la batalla leones y tigres y sorras y buitres, gabilanes y gatos de monte. Y ancí sus desendientes hasta oy se llaman poma [león], otorongo [jaguar], atoc [zorro], condor, anca [gavilán], usco [gato montés], y biento, acapana [celajes], páxaro, uayanay [papagayo]; colebra, machacuay; serpiente, amaro. Y ací se llamaron de otros animales sus nombres y armas que trayya sus antepasados; los ganaron en la batalla que ellos tubieron el más estimado nombre de señor fue poma, guaman [halcón], anca, condor, acapana, guayanay, curi [oro], cullque [plata], como parese hasta oy. (Guamán Poma 1980[1615], p. 52)

Lo primero a destacar es que el pasaje no solo relaciona el conflicto con la crueldad, la traición y la muerte, sino también con la riqueza y la vida. Esta ambigüedad tiene paralelos en otras expresiones vinculadas al conflicto (p. ej., léxicos, iconografía, armas y trofeos), que lo asocian también con el matrimonio, la complementación, la fertilidad y la renovación de la vida (Topic y Topic 1997). El segundo punto que interesa subrayar se refiere a la memoria de los antiguos guerreros y sus logros. Gracias a los méritos ganados en combate, los awca runa alcanzaron posiciones de autoridad (capitanes y príncipes) y se convirtieron en fundadores de linajes, es decir, antepasados. Sin embargo, no se recuerdan sus nombres sino los de los animales tutelares y armas que encarnaron en batalla, borrando así su individualidad de la memoria. De acuerdo con la orientación corporativa notada a partir de otros aspectos del registro material, fueron los ancestros, la colectividad de sus descendientes y las armas devenidas en emblemas, quienes atesoraron el poder y la riqueza nacidos de aquella era de conflictos. 


\section{BIBLIOGRAPHY}

Arkush, E.(2006). Collapse, conflict, conquest: The transformation of warfare in the Late Prehispanic Andean Highlands. En The Archaeology of Warfare: Prehistories of Raiding and Conquest. Gainesville:University Press of Florida.

Arkush, E.(2008). War, chronology, and causality in the Titicaca Basin. Latin American Antiquity, 19, 339-373.

Arkush, E. y Stanish, Ch.(2005). Interpreting conflict in the ancient Andes: Implications for the Archaeology of Warfare. Current Anthropology, 46, 3-28.

Arkush, E. y Tung, T. A. (2013). Patterns of war in the Andes from the Archaic to the Late Horizon: insights from settlement patterns and cranial trauma. Journal of Archaeological Research, 21(4), 307-369.

Balesta, B. y Wynveldt, F.(2010). La Loma de Ichanga: visibilidad, defensibilidad y abandono en el valle de Hualfín (Depto. de Belén, Prov. de Catamarca, Argentina). Revista Española de Antropología Americana, 40, 53-71.

Berenguer, J.(2006). Señales en la cabeza: Los tocados de Wirakocha en el norte de Chile.Santiago: Museo Chileno de Arte Precolombino.

Carneiro, R. L. (1970). A Theory of the Origin of theState.Science,169, 733-738.

Casanova, E.(1936). La Quebrada de Humahuaca. Historia de la Nación Argentina, Volumen 1, 207-249. Buenos Aires: Junta de Historia y Numismática Americana.

Cieza de León, P.(1984 [1553]). Crónica del Perú. Lima: Pontificia Universidad Católica del PerúAcademia Nacional de la Historia(3 vol.).

Earle, T. K. (1997). How chiefs come to power. Stanford: Stanford University Press.

Ferguson, R. B.(1995). Yanomami warfare. Santa Fe: School of American Research Press.

Gheggi, M. S. y Seldes, V. (2012). Evidencias bioarqueológicas de conflicto ca. 1000-1432 AD en la Quebrada de Humahuaca y el Valle Calchaquí. Intersecciones,13, 103-115.

Guamán Poma de Ayala, F.(1980 [1615]).Nueva Crónica y Buen Gobierno. México: Siglo XXI.

Haas, J.(2001).Warfare and the evolution of culture. En G. Feinman y T. D. Price (Eds.),Archaeology at the Millennium(pp. 329-350). New York: Springer.

Keeley, L. H. (1996). War before civilization. New York: Oxford University Press.

LeBlanc, S. (1999). Prehistoric warfare in the American southwest. Salt Lake City: The University of Utah Press.

Llagostera, A.(2010). Retomando los límites y las limitaciones del "archipiélago vertical". Chungara Revista de Antropología Chilena, 42:283-295.

Madrazzo, G. y Ottonello, M. (1966). Tipos de instalación prehispánica en la región de la puna y su borde. Monografías, 1. Olavarría: Museo Etnográfico Municipal "Dámaso Arce".

Morales, M.S., Christie, D.A., Villalba, R., Argollo, J., Pacajes, J., Silva, J.S., Alvarez, C.A., Llancabure, J.C. y Soliz Gamboa, C.C. (2012).Precipitation changes in the South American Altiplano since $1300 \mathrm{AD}$ reconstructed by tree-rings. Climate of the past, 8:653-666. 
Nielsen, A. E. (2001). Evolución social en Quebrada de Humahuaca (AD 700-1536). En E. Berberián y A. Nielsen (Eds.), Historia argentina prehispánica (Vol. I, pp. 171-264). Córdoba: Editorial Brujas.

Nielsen, A. E. (2002). Asentamientos, conflicto y cambio social en el Altiplano de Lípez (Potosí, Bolivia). Revista Española de Antropología Americana, 32:179-205.

Nielsen, A. E. (2003).La edad de los auca runa en la Quebrada de Humahuaca (Jujuy, Argentina). Memoria Americana, 11: 73-109.

Nielsen, A. E. (2006). Plazas para los antepasados: descentralización y poder corporativo en las formaciones políticas preincaicas de los Andes circumpuneños. Estudios Atacameños,31:63-89.

Nielsen, A. E. (2007). Armas significantes: tramas culturales, guerra y cambio social en el sur andino prehispánico. Boletín del Museo Chileno de Arte Precolombino,12(1), 9-41. Santiago de Chile.

Núñez Regueiro, V.(1974). Conceptos instrumentales y marco teórico en relación al análisis del desarrollo cultural del Noroeste Argentino. Revista del Instituto de Antropología, V, 169-190.

Córdoba.

Platt, T.(1987). Entre Ch'axway Muxsa: para una historia del pensamiento político aymara. En Tres Reflexiones sobre el Pensamiento Andino (pp. 61-132). La Paz: Hisbol.

Redmond, E. M. (1994). Tribal and chiefly warfare in South America. Memoirs of the Museum of Anthropology, 28.Ann Arbor: University of Michigan.

Rothammer, F. y Santoro, C.(2001). El desarrollo cultural en el valle de Azapa, extremo norte de Chile y su vinculación con los desplazamientos poblacionales altiplánicos. Latin American Antiquity, 12, 59-66.

Ruiz, M. y Albeck, M. E. (1997). El fenómeno pukara visto desde la puna jujeña. EstudiosAtacameños ,12, 83-95.

Schiappacasse, V., Castro. V. y Niemeyer, H.(1989). Los desarrollos regionales en el Norte Grande (1000-1400 d.C.). En J. Hidalgo, V. Schiappacasse y H. Niemeyer (Eds.), Culturas de Chile: Prehistoria(pp.181-220). Santiago:Andrés Bello.

Topic, J. y Topic, T.(1997). Hacia una comprensión conceptual de la guerra andina. En R. Gabai y J. Flores Espinoza(Eds.), Arqueología, antropología e historia en los Andes: homenaje a María Rostworowski (pp. 567-590). Lima: Instituto de Estudios Peruanos,.

Topic, J. y Topic, T. (2009).Variation in the practice of prehispanic warfare on the north coast of Peru. En A. E. Nielsen y W. H. Walker (Eds.), Warfare in cultural context: Practice, agency, and the archaeology of violence (pp. 17-55). Tucson: University of Arizona Press.

Torres-Rouff, Ch., Costa-Junqueira M. A. y Llagostera,A.(2005). Violence in times of change: The Late Intermediate Period in San Pedro de Atacama. Chungará, 37:75-83.

Wiessner, P. (2009). Warfare and political complexity in an egalitarian society: An Ethnohistorical Example. En A. E. Nielsen y W. H. Walker (Eds.), Warfare in cultural context: Practice, agency, and the archaeology of violence (pp. 165-189). Tucson: University of Arizona Press.

Wiessner, P. y Tumu, A. (1998). Historical vines: Enganetworks of exchange, ritual, and warfare in Papua New Guinea. Washington: Smithsonian Institution.

Williams, V. (2014a). Quebradas altas del Calchaquí medio. Dinámica social durante el Período de Desarrollos Regionales y el Tawantinsuyu. En M. E. Albeck, M. B. Cremonte y M. Ruiz (Eds.), Las tierras altas del area centro sur andina entre 1000 y 1600 d.C. TANOA II (pp. 159-192). San Salvador de Jujuy: EdiUnju. 
Williams, V.(2014b). Sociedades prehispánicas tardías en el noroeste argentino. Una aproximación a trayectorias históricas en el Valle Calchaquí durante el Tawantinsuyu. En Ocupación inka y dinámicas regionales en los Andes (Siglos XV-XVII). La Paz:Plural.

Wynveldt, F. y Balesta, B. (2009). Paisaje socio-político y beligerancia en el Valle de Hualfín. Antípoda, 8, 143-168.

\section{NOTES}

1. No hay datos suficientes para evaluar la situación de los valles boscosos del piedemonte andino oriental.

2. Cabe aclarar que, aunque existen asentamientos más vulnerables, hay gran cantidad de sitios fortificados en el sur andino. Desde el lago Titicaca hasta Lípez, por ejemplo, los pukaras del altiplano poseen murallas defensivas concéntricas con accesos controlados, troneras y parapetos y se encuentran frecuentemente equipados con abundantes proyectiles en previsión de asaltos (Arkush y Stanish 2005,Nielsen 2002).

\section{AUTHOR}

\section{AXEL E. NIELSEN}

CONICET (Consejo Nacional de Investigaciones Científicas y Técnicas) - Instituto Nacional de Antropología y Pensamiento Latinoamericano, Argentina.

Correo electrónico: axelnielsen@gmail.com 